\title{
Anti-inflammation effects of picroside 2 in cerebral ischemic injury rats
}

Yunliang Guo*1, Xinying Xu1', Qin Li1,2, Zhen Li1,3 and Fang Du

\begin{abstract}
Background: Excitatory amino acid toxicity, oxidative stress, intracellular calcium overload, as well as inflammation and apoptosis are involved in the pathological process after cerebral ischemic reperfusion injury. Picrodide 2 could inhibit neuronal apoptosis and play anti-oxidant and anti-inflammation role in cerebral ischemia/reperfusion injuries, but the exact mechanism is not very clear. This study aims to explore the anti-inflammation mechanism of picroside 2 in cerebral ischemic reperfusion injury in rats.
\end{abstract}

Methods: The middle cerebral artery occlusion reperfusion models were established with intraluminal thread methods in 90 adult healthy female Wistar rats. Picroside 2 and salvianic acid A sodium were respectively injected from tail vein at the dosage of $10 \mathrm{mg} / \mathrm{kg}$ for treatment. The neurobehavioral function was evaluated with Bederson's test and the cerebral infarction volume was observed with tetrazolium chloride (TTC) staining. The apoptotic cells were counted by in situ terminal deoxynucleotidyl transferase-mediated biotinylated deoxyuridine triphosphate nick end labeling (TUNEL) assay. The immunohistochemistry stain was used to determine the expressions of toll-like receptor 4 (TLR4), nuclear transcription factor KB (NFKB) and tumor necrosis factor a (TNFa). The concentrations of TLR4, NFKB and TNFa in brain tissue were determined by enzyme linked immunosorbent assay (ELISA).

Results: After cerebral ischemic reperfusion, the rats showed neurobehavioral function deficit and cerebral infarction in the ischemic hemisphere. The number of apoptotic cells, the expressions and the concentrations in brain tissue of TLR4, NFKB and TNFa in ischemia control group increased significantly than those in the sham operative group $(P<$ 0.01). Compared with the ischemia control group, the neurobehavioral scores, the infarction volumes, the apoptotic cells, the expressions and concentrations in brain tissue of TLR4, NFKB and TNFa were obviously decreased both in the picroside 2 and salvianic acid A sodium groups $(P<0.01)$. There was no statistical difference between the two treatment groups in above indexes $(P>0.05)$.

Conclusions: Picroside 2 could down-regulate the expressions of TLR4, NFKB and TNFa to inhibit apoptosis and inflammation induced by cerebral ischemic reperfusion injury and improve the neurobehavioral function of rats.

\section{Background}

Excitatory amino acid toxicity, oxidative stress, intracellular calcium overload, as well as inflammation and apoptosis, are involved in the pathological process after cerebral ischemic reperfusion injury[1]. Among these, the inflammatory cytokines activate nuclear transcription factor $\kappa B(\mathrm{NF} \kappa \mathrm{B})$ through toll-like receptor 4 (TLR4)$\mathrm{NF \kappa B}$ signal transduction pathways, promote target gene activation, such as tumor necrosis factor $\alpha$ (TNF $\alpha)$, interleukin (IL), intercellular adhesion molecule (ICAM), and

\footnotetext{
* Correspondence: guoqdsd@yahoo.com.cn

1 Institute of Cerebrovascular Diseases, Affiliated Hospital of Qingdao University Medical College, Qingdao 266003, China

Full list of author information is available at the end of the article
}

ultimately induce neuronal apoptosis[2,3]. Picrorhiza scrophulariflora belongs to the plant family composed of picroside 1, 2 and 3, of which picroside 2 is one of the most effective components extracted from the dried rhizome and roots of Picrorhiza kurrooa Royle ex Benth[4] and Picrorhiza scrophulariae flora Pennell [5]. It has been traditionally used to treat disorders of the liver, upper respiratory tract diseases, dyspepsia, chronic diarrhea and scorpion sting $[5,6]$. Current researches on picroside 2 are focused on its neuroprotective[7], anti-apoptotic, anti-cholestatic, anti-oxidant, anti-inflammation, immunemodulating activities $[8,9]$. It has been confirmed that picroside 2 could enhance nerve growth factor-induced 
PC12 cell axon growth, reduce $\mathrm{H}_{2} \mathrm{O}_{2}$ induced PC12 cell damage and improve cell survival in vitro [10-13]. Animal experiments showed that the extractive of Picrorhizae could inhibit cell apoptosis in ischemic penumbra and improve neurobehavioral function in middle cerebral artery occlusion and reperfusion $(\mathrm{MCAO} / \mathrm{R})$ rats[14,15]. Our previous experiments[16-19] indicated that picroside 2 could inhibit the expressions of inducible nitric oxide synthase (iNOS), nuclear transcription factor $\kappa B$ $(\mathrm{NF} \kappa \mathrm{B})$ and inhibitor of NFkB (ІкB), and reduce the expressions of Caspase-3 and poly ADP-ribose polymerase (PARP) to inhibit the neuronal apoptosis and thus improve the neurobehavioral function of rats with cerebral ischemia reperfusion injury. However, it is poorly understood that how many ingredients there are in the extract Picrorhizae and which one can play physiological role. In the study, we established the experimental $\mathrm{MCAO} / \mathrm{R}$ model to investigate the effects of picroside 2 via tail vein injection on TLR4-NFKB signal transduction pathway and cell apoptosis, and to explore its antiinflammatory mechanism in cerebral ischemic reperfusion injury.

\section{Methods}

\section{Establishment of animal models}

The total of 90 adult female Wistar rats, weight 230 - 250 g, SPF grade, were granted by Qingdao Laboratory Animal Center (SCXK (LU) 20030010). The guidance suggestions for care of laboratory animals was followed according to the Guidelines for caring for experimental animals published by the Ministry of Science and Technology of the People's Republic of China. All animals were reared in the laboratory environment, allowed free access to food and water in room temperature and humidity-controlled housing with natural illumination for a week, and fasted $12 \mathrm{~h}$ before operation. Fifteen rats were randomly selected as a sham operated group (SO), and the rest 75 rats were subjected to the experimental middle cerebral artery occlusion $2 \mathrm{~h}$ and reperfusion $22 \mathrm{~h}$ $(\mathrm{MCAO} / \mathrm{R})$ models with intraluminal monofilament suture from left external-internal carotid artery [20,21]. At $2 \mathrm{~h}$ after the operation, those with left Horner sign, right anterior limb flexible or circling towards right, were considered as symbols of successful models. Core body temperature was monitored with a rectal probe and maintained at $36^{\circ} \mathrm{C}-37^{\circ} \mathrm{C}$ using a homeothermic blanket control unit (Qingdao Apparatus, China) during and after the surgery operation. As a result, 45 successful models, as the candidate, were randomly divided into a ischemia control group (IC, $\mathrm{n}=15$ ), a salvianic acid A sodium group $(\mathrm{SA}, \mathrm{n}=15)$ and a picroside treated group $(\mathrm{PT}, \mathrm{n}=$ 15) according to drug administration. Rats in the $\mathrm{SO}$ group were experimented with the same surgical procedure but the monofilament was advanced only about 10 $\mathrm{mm}$ and immediately withdrew. The 30 dead or unsuccessfully occluded rats were excluded from this experiment.

\section{Intervention study}

According to the Pharmacopoeia of the People's Republic of China, the molecular formula of picroside 2 is $\mathrm{C}_{23} \mathrm{H}_{28} \mathrm{O}_{13}$, molecular weight is 512.48 ; the molecular formula of salvianic acid A sodium is $\mathrm{C}_{9} \mathrm{H}_{9} \mathrm{O}_{5} \mathrm{Na}$, molecular weight is 221.17. The chemical structure formulae[22] are shown as figure 1 :

Picroside 2 (Tianjin Kuiqing Medical Technology Co. Ltd., CAS No: 39012-20-9, purity > 98\%), as a treatment

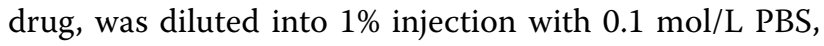
and salvianic acid A sodium (Shanghai Huyun Medical Technology Co. Ltd., CAS No: 23028-17-3, purity > 98\%) was also diluted into $1 \%$ solution as a positive control drug. According to Xiao's report [23], the rats in PT group were administrated picroside $2(10 \mathrm{mg} / \mathrm{kg}) 250 \mu \mathrm{l}$ via tail vein at ischemia $2 \mathrm{~h}$ prior to reperfusion with a micro-syringe. Rats in SA group were given salvianic acid A sodium $(10 \mathrm{mg} / \mathrm{kg}) 250 \mu \mathrm{l}$, while those in IC group and SO group were simultaneously suffered $0.1 \mathrm{~mol} / \mathrm{L} \mathrm{PBS}$ $250 \mu \mathrm{l}$.

\section{Neurobehavioral function assessment}

All animal neurobehavioral tests were performed at ischemia $2 \mathrm{~h}$ and reperfusion $22 \mathrm{~h}$ by an investigator who was blinded to the experiment according to the standard of Bederson's report[24]. 0 score: no neurological functional

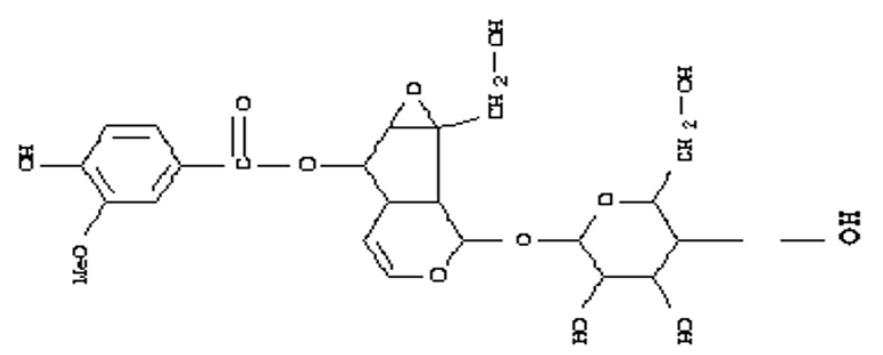<smiles>CC(=O)C(O)Cc1ccc(O)c(O)c1</smiles>

Figure 1 The chemical structure formula of picroside 2 (left) and salvianic acid A sodium (right). 
impairment; 1 score: any part of forepaw flexed (positive for tail suspension test) without other abnormal signs; 2 scores: lateral pushing resistance ability decreased (positive for lateral pushing experiment), accompanied with forepaw flexion without circling tendency; 3 scores: same behavior as those for 2 scores, in addition to spontaneous rotation (circling around paralyzed limbs during free activity). The higher the score is gotten, the worse the neurobehavioral dysfunction is appeared, vice versa.

\section{TTC staining}

To determine the infarction volume, five rats in each group were deeply anesthetized by $10 \%$ chloral hydrate (300 mg/kg) and decapitated at given time after MCAO/ $\mathrm{R}$. The brain tissue was removed and successivelly sliced into $2.0 \mathrm{~mm}$-thick coronal sections. The total of five brain slices were incubated in $2 \%$ TTC solution for $10 \mathrm{~min}$ at $37^{\circ} \mathrm{C}$ and then transferred into $4 \%$ formaldehyde solution for fixation. The normal brain tissue appeared uniform red while the infarction region showed white. The infarction volumes were calculated in a blinded manner with Adobe PhotoShop CS analysis system. The data were expressed as the percentage of the infarction volume/the ipsilateral hemisphere volume (\%) at the coronal section of optic chiasma.

\section{Neuronal apoptosis assay}

Five rats in each group were deeply anesthetized by $10 \%$ chloral hydrate $(300 \mathrm{mg} / \mathrm{kg})$, reperfused with sodium chloride and $4 \%$ formaldehyde $200 \mathrm{ml}$ from the heart into the aorta, and then decapitated at given time. Brain samples were chosen from frontal fontanelle $2 \mathrm{~mm}$ to occipital fontanelle $4 \mathrm{~mm}$ by a stereotaxic point, post-fixed in $4 \%$ formaldehyde for $2 \mathrm{~h}$, dehydrated in alcohol gradually, hyalinized by dimethylbenzene, embedded in paraffin, then sectioned at a thickness of $5 \mu \mathrm{m}$, adhered to the sections prepared with poly-L-Lysine, and finally stored at $4^{\circ} \mathrm{C}$. To detect cell apoptosis, TUNEL staining was performed according to the protocol of DendEnd Fluorometric TUNEL Detection System (Santa Cruz Co. Ltd.). Coronal paraffin sections described as above were deparaffinaged by dimethylbenzene, hydrated by gradient ethanol and washed by distilled water. Some sections added DNase I at a dose of $1 \mu \mathrm{g} / \mathrm{ml}$ were regarded as positive control sample, and those treated without $\mathrm{TdT}$ were the negative ones. Under a 400-fold immunofluorescent microscope (wavelength $488 \mathrm{~nm}$ ), the apoptotic cells were appeared yellow-green fluorescence in nucleus, and averaged in four random views in cortex and striatum, respectively.

\section{Immunohistochemical staining}

Rabbit anti-rat TLR4, NFKB and TNF $\alpha$ moloclonal antibody (1:100) were purchased from Santa Cruz Co. Ltd.
Strept-avidin-biotin complex (SABC) immunohistochemistry kit, diaminobenzidine (DAB) dye were granted by Bostor biological company in Wuhan China. Paraffinembedded sections were deparaffinaged in dimethylbenzene, hydrated successively in gradient ethanol, restored antigen twice in a microwave oven. Immunohistochemical procedures were performed strictly according to the guidance of manufacture. Under a microscope, those with brown granules in cytoplasm or nucleus were considered as positive cells. And the slides added $0.1 \mathrm{mmol} / \mathrm{L}$ PBS (containing 1:200 non-immunity animal serum) instead of primary antibody had no response. Four serial sections were chosen from each experimental rat and observed randomly four views at cortex and striatum under a 400-fold microscope. Absorbance values $(A)$ of each view was detected by a LEICA Qwin microgramme analytical system (Leica company).

\section{Enzyme linked immunosorbent assay (ELISA)}

Rat TLR4 (No. E02T0013), NFkB p65 (No. E02N0014) and TNF $\alpha$ (No. E02T0012) ELISA kits were purchased from Blue Gene Co. Ltd. Five rats in each group were deeply anesthetized and decapitated at given time after $\mathrm{MCAO} / \mathrm{R}$. The ischemic hemisphere tissue $(0.5 \mathrm{~g})$ was quickly removed and ground fully into brain tissue homogenate. Then added normal sodium $500 \mu \mathrm{l}$, mixed well and centrifugalized for 10 minutes at $12000 \mathrm{r} / \mathrm{min}$. The upper limpid liquid was collected and stored at $-20^{\circ} \mathrm{C}$ (Avoid repeated freeze-thaw cycles). Prepare all standards before starting assay procedure. First, secure the desired number of coated wells in the holder, then add 50 $\mu \mathrm{l}$ of standards or samples to the appropriate well of the antibody pre-coated microtiter plate. Add $100 \mu \mathrm{l}$ of conjugate to each well. Mix well, cover and incubate for $1 \mathrm{~h}$ at $37^{\circ} \mathrm{C}$. Wash the microtiter plate 5 times using distilled or de-ionized water. Add $50 \mu \mathrm{l}$ substrate A \& B to each well. Cover and incubate for 15 minutes at $25^{\circ} \mathrm{C}$. Add $50 \mu$ l of stop solution to each well. Mix well and calculate the mean absorbance value $A_{450}$ for each set of reference standards and samples. The standard density is a $X$, the $\mathrm{B} / \mathrm{B} 0$ is a $\mathrm{Y}$, sitting to mark the paper in the logit-log up draw a standard curve. According to the B/B0 that need to be measured the sample can from sit to mark the density value that the paper looks up the sample up. The sensitivity by this assay is $1.0 \mathrm{pg} / \mathrm{ml}$ (TLR4 and TNF $\alpha$ ) and $1.0 \mathrm{ng} / \mathrm{ml}(\mathrm{NF} \kappa \mathrm{B})$.

\section{Statistical Analysis}

SPSS11.5 software was used for statistical analysis. Data were expressed as mean \pm standard error $(\bar{x} \pm \mathrm{s})$. Multigroup comparison was made by analysis of variance (ANOVA) and Student's test, and two-group comparison 
by $t$-test. Values were considered to be significant when $P$ is less than 0.05 .

\section{Results}

\section{Neurobehavioral dysfunction score}

There was no neurobehavioral dysfunction symptom in rats of the $\mathrm{SO}$ group, whose Bederson's score got 0 points. After cerebral ischemia/reperfusion injury, all animals showed neurobehavioral dysfunction. The Bederson's scores both in the SA group and PT group were obviously lower than that in the IC group $(F=24.90, q=8.38-8.88$, $P<0.01)$. The scores in PT group is slightly lower than that in SA group, but no significant difference was found between these two groups $(t=0.50, P>0.05)$. Shown as table 1.

\section{The cerebral infarction volume}

By TTC stain, no cerebral ischemia infarction was shown in the brain slices of the SO group, while infarction lesion almost appeared in all the experimental rats after cerebral ischemic reperfusion injury. The volume of cerebral infarction in the SA group and PT group were significantly lower than that in the IC group $(F=30.76, q=$ 8.66-10.33, $P<0.01)$, but there is no statistical difference between the SA group and PT group $(t=1.66, P>0.05)$. Shown as table 1 and figure 2 .

\section{Neuronal apoptosis}

A few apoptotic cells were scattered in the cortex and the striatum in SO group rats. Apoptotic cells were significantly increased in the IC group. As we expected, the number of apoptosis both in the SA group and PT group were obviously low compared with the IC group $(F=$ 29.05, $q=4.03-12.84, P<0.01)$, but no difference existed between the SA group and PT group $(t=0.49, P>0.05)$. Shown as table 1 and figure 3.

\section{The expressions of TLR4, NFKB and TNFa}

With the help of immunohistochemistry, we found that there were no region differences between the cortex and the striatum. Thus we calculated the absorbance values $(A)$ in four confirmed views instead of the random ones in each brain slice.

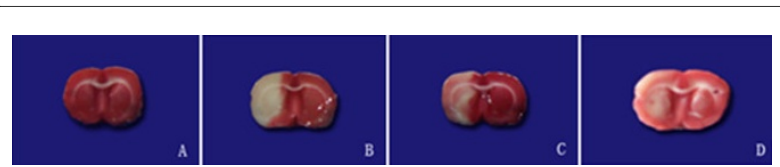

Figure 2 The cerebral infarction volume shown by TTC stain (A. SO group, B. IC group, C. SA group, D. PT group).

Few TLR4 positive cells with light yellow granules could be seen in the cortex and the striatum in SO group rats, and its expression was very weak. TLR4 expression in IC group was significantly elevated along with the increased $A$ value as compared with the SO group rats; After the drug administration, the $A$ values in the SA group and PT group were obviously decreased in contrast to the IC group rats $(F=4.33, q=2.95-4.92, P<0.05)$. Although the $A$ value in the SA group was slightly lower than that in the PT group, there was no significant differences between the two treatment groups, suggesting that picroside 2 could inhibit the expression of TLR4 protein and play neuroprotective effects as same as salvianic acid A sodium $(t=1.31, P>0.05)$. Shown as table 2 and figure 4 .

Weak NFKB expression were shown in the cortex and the striatum in $\mathrm{SO}$ group rats. The number of NFkB positive cells rapidly increased, and its A value was significantly higher than that in the SO group; the expression of $\mathrm{NF \kappa B}$ both in the SA group and PT group were apparently lower than that in the IC group $(\mathrm{F}=14.25, \mathrm{q}=6.24$ $8.17, \mathrm{P}<0.01)$, but there was no difference between the SA group and PT group as shown in table 2 and figure $5(\mathrm{t}$ $=0.64, \mathrm{P}>0.05$ ).

Ditto, the TNF $\alpha$ expression was very weak in the cortex and the striatum in SO group rats. TNF $\alpha$ positive cells was clearly increased in the IC group, and its $A$ value was significantly higher than that in the SO group; the $A$ values in the SA and PT groups were obviously low compared with the IC group $(F=6.39, q=3.42-6.03, P<0.05)$, and there was no difference between the SA group and the PT group, too $(t=0.81, P>0.05)$. Shown as table 2 and figure 6.

The concentrations in brain tissue of TLR4, NFKB and TNFa The concentrations in brain tissue of TLR4, NFKB and TNF $\alpha$ were lowly in SO group rats, and increased signifi-

Table 1: Bederson's score, infarction volume and apoptotic cells ( $\bar{x} \pm s)$

\begin{tabular}{cccc}
\hline Groups & Bederson's score $(\mathbf{n}=\mathbf{1 5})$ & Infarction volume( $\mathbf{n}=\mathbf{5})$ & Apoptotic cells $(\mathbf{n}=\mathbf{5})$ \\
\hline SO group & $0.00 \pm 0.00$ & $0.00 \pm 0.00$ & $3.53 \pm 1.13$ \\
IC group & $2.17 \pm 0.35^{\Delta}$ & $77.32 \pm 3.06^{\Delta}$ & $16.62 \pm 3.25^{\Delta}$ \\
SA group & $1.33 \pm 0.43^{*}$ & $70.11 \pm 3.13^{*}$ & $8.13 \pm 2.15^{*}$ \\
PT group & $1.28 \pm 0.38^{* \#}$ & $68.73 \pm 3.46^{* \#}$ & $7.64 \pm 2.08^{* \#}$ \\
\hline
\end{tabular}

$\Delta P<0.01$, vs the SO group; ${ }^{*} P<0.01$, vs the IC group; ${ }^{\#} P>0.05$, vs the SA group. 


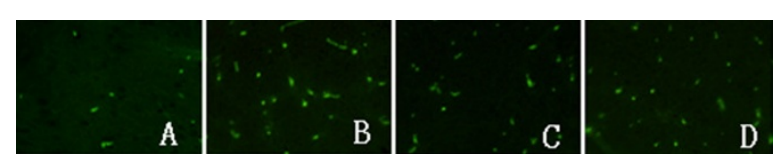

Figure 3 Apoptosis in cortex shown by TUNEL $\times 200$ (A. SO group, B. IC group, C. SA group, D. PT group)

cantly in the IC group rats. In the SA group and PT group, the concentrations of TLR4 $(F=79.42, q=7.20$ 21.43, $P<0.01)$, NFkB $(F=111.25, q=7.98-25.27, P<$ $0.01)$ and TNF $\alpha(F=95.29, q=6.89-23.27, P<0.01)$ were obviously lower than those in the IC group, but there was no difference between the SA group and PT group $(t=$ $170,1.01,1.69, P>0.05)$. Shown as table 3.

\section{Discussion}

TLR4 is a member of signal transduction family, which combines with the adapter protein MyD88 and then with interleukin-associated kinase (IRAKs) to cause NFkB translocation, to enable the innate immune and inflammatory responses related gene transcription, to activate the TLR4-MyD88 dependent signal transduction pathway and to induce inflammatory response[1]. Caso et al [2] found that the cerebral infarction volume in TLR4 deficient rats was smaller than that in normal TLR4 animals after middle cerebral artery occlusion and reperfusion. Hua et al [25] proved that cerebral infarction size in TLR4 knockout mice was also smaller than the wild-type ones, and its neurological deficit score was lower accordingly. The studies of Yang et al [26] showed that the TLR4 positive lymphomonocytes in blood circulation significantly elevated in patients with acute cerebral infarction as compared with that in the control group and the patients with transient ischemic attack, and the expression levels of TLR4 mRNA and TNF $\alpha$ in serum were consistent with IL6. From the data of correlation analysis, they further found that TLR4 and serum cytokine expression levels were closely related to the severity of stroke. Thus they speculated it is TLR4 that activates the downstream signaling protein, evokes the gene transcription associated with the encoding and inflammation-related

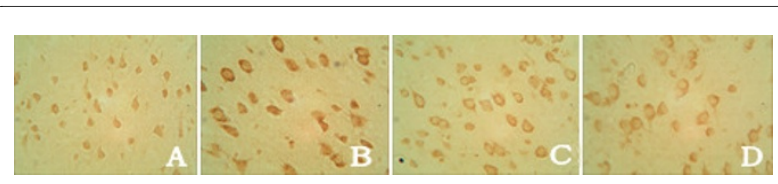

Figure 4 TLR4 expression in cortex shown by $S A B C \times 400$ (A. SO group, B. IC group, C. SA group, D. PT group)

factor, induces and aggravates the inflammatory response.

$\mathrm{NF} \kappa \mathrm{B}$ is an important nuclear transcription factor in eukaryotic cells, which exists in almost all the cells. It is located downstream of TLR signaling pathway, regulated by TLRs and involved in immune response and the processes of cell proliferation and differentiation. Cerebral ischemic reperfusion injury not only promotes the innate immune response of the immune system, but also activates TLR4-mediated signal transduction pathway to rapidly translocate $\mathrm{NF}_{\mathrm{K} B}$ from cytoplasm into nucleus, and combines with specific DNA sequence to stimulate downstream-associated factor (TNF $\alpha$ )-induced inflammation response and ischemia neuronal apoptosis[27]. With the application of MCAO models, Lou et al [28] confirmed that the increased TNF $\alpha$ protein and its mRNA expression and NFKB DNA-binding-activity could reduce the survival number of spinal cells in the hippocampal CA1 area, suggesting that NFKB activation can lead to neuronal apoptosis in inflammation following cerebral ischemic reperfusion injury. It will become a new treatment target to reduce cerebral ischemic reperfusion injury through efficiently decreasing TNF $\alpha$ protein and mRNA expression and inhibiting NFkB DNA bindingactivity. Liu et al [29] reperted that the expression of $\mathrm{NF} \kappa \mathrm{B}$ enhanced in cytoplasmic or nuclear in brain slices, and the use of Oxymatrine decreased the NFKB expression and the infarction lesions in rats with permanent middle cerebral artery occlusion compared with the sham operation group. Webster et al [30] found that hypothermy could attenuate the NFKB inhibition factor (I KB) phosphorylation and ІкB suppressor kinase, and decrease the expression of $\mathrm{NFKB}$, thereby protect neurons in penumbra against focal ischemic reperfusion damage. The data of Zhang et al [31] have demonstrated that the

Table 2: Absorbance values of TLR4, NFKB and TNFa expressions ( $\bar{x} \pm s$ )

\begin{tabular}{ccccc}
\hline Groups & $\mathbf{n}$ & TLR4 & NFKB & TNFa \\
\hline SO group & 5 & $0.16 \pm 0.09$ & $0.14 \pm 0.07$ & $0.14 \pm 0.08$ \\
IC group & 5 & $0.46 \pm 0.18^{\Delta}$ & $0.65 \pm 0.21^{\Delta}$ & $0.51 \pm 0.17^{\Delta}$ \\
SA group & 5 & $0.28 \pm 0.13^{*}$ & $0.26 \pm 0.15^{*}$ & $0.30 \pm 0.16^{*}$ \\
PT group & 5 & $0.24 \pm 0.13^{* \#}$ & $0.22 \pm 0.08^{* \#}$ & $0.25 \pm 0.12^{* \#}$ \\
\hline
\end{tabular}

$\Delta P<0.01$, vs the SO group; ${ }^{*} P<0.01$, vs the IC group; ${ }^{P}>0.05$, vs the SA group. 


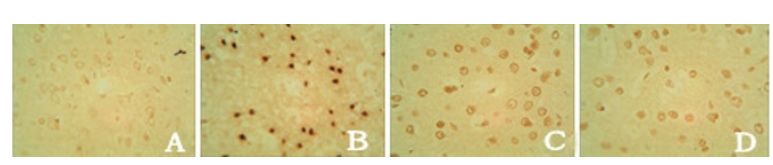

Figure $5 \mathrm{NFkB}$ expression in cortex shown by SABC $\times 400$ (A. SO group, B. IC group, C. SA group, D. PT group)

intracerebroventricular administration of prostaglandin after cerebral ischemia could inhibit the expression of subunit NFKB and play the protection role in ischemia neurons. These results intimate that the inhibition of $\mathrm{NF \kappa B}$ expression to reduce apoptosis may be one of the mechanisms to improve the brain ischemic reperfusion injury. Our previous researches proved that picroside 2 could inhibit the expressions of iNOS [16], NFKB and IкB[17], and reduce the expressions of Caspase-3, PARP $[18]$ and to inhibit the neuronal apoptosis and thus improve the neurological function of rats with cerebral ischemia reperfusion injury[19]. It also reduced brain tissue edema and the aquaporin-4 (AQP4) expression, and the best therapeutic time window was $1 \mathrm{~h}$ after cerebral ischemic reperfusion [32].

This experiment showed that slight expressions of TLR4, NFKB and TNF $\alpha$ could be seen in the cortex and the striatum in the SO group, and their concentrations in brain tissue were very lowly. After cerebral ischemia $2 \mathrm{~h}$ and reperfusion $22 \mathrm{~h}$, the expressions of these proteins, as well as the TUNEL-positive cells and concentrations in brain tissue increased significantly in contrast to the $\mathrm{SO}$ group. The data imply that the activation of TLR4 and its downstream NFKB inflammatory factor induce neuronal apoptosis and cerebral ischemic reperfusion injury. In this experiment, $\mathrm{NF}_{\kappa} \mathrm{B}$ protein inactively expressed in cytoplasm in the SO group. After cerebral ischemic reperfusion, it mainly located in nuclear, suggesting that the activation of $\mathrm{NFKB}$ into nucleus promote the expression of the downstream target genes TNF $\alpha$, be involved in inflammatory response and induce neuronal apoptosis. Accordingly, the experimental animals appeared cerebral infarction lesions, abnormal morphology and the increased neuronal apoptosis in ischemic ipsilateral, and

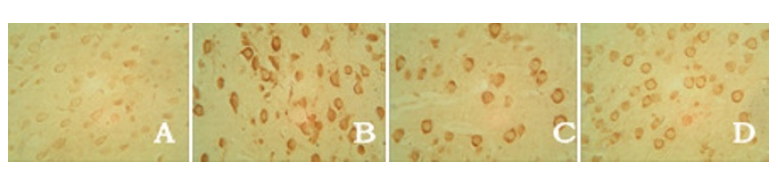

Figure 6 TNFa expression in cortex shown by SABC $\times 400$ (A. SO group, B. IC group, C. SA group, D. PT group).

neurobehavioral dysfunction in contralateral hemisphere. We also found that the injection of picroside 2 and salvianic acid A sodium via tail vein significantly reduced the amount of apoptotic cells, the concentrations in brain tissue and the expressions of TLR4, NFKB and TNF $\alpha$ proteins in the cortex and the striatum in $\mathrm{MCAO} / \mathrm{R}$ rats, together with the decreased infarction size and the improvement of cellular structure and neurobehavioral function of rats. In short, the neuroprotective effect of picroside 2 and salvianic acid A sodium against cerebral ischemic reperfusion injury might be performed by inhibiting TLR4-NFkB signal transduction pathway to reduce inflammatory response-induced apoptosis. However, the concrete differences between the two Chinese drugs needs to further elucidated.

\section{Conclusion}

Picroside 2 could down-regulate the expressions of TLR4, $\mathrm{NF} K \mathrm{~B}$ and TNF $\alpha$ to inhibit apoptosis and inflammation induced by cerebral ischemic reperfusion injury and improve the neurobehavioral function of rats.

\section{List of abbreviations used}

MCAO/R: middle cerebral artery occlusion/reperfusion; SO group: sham operated group; IC group: ischemia control group; SA group: salvianic acid A sodium group; PT group: picroside treated group; TTC: tetrazolium chloride; TLR4: toll-like receptor 4; NFKB: nuclear transcription factor $\kappa B$; TNF $\alpha$ : tumor necrosis factor $\alpha$; TUNEL: terminal deoxynucleotidyl transferase-mediated biotinylated deoxyuridine triphosphate nick end labelling; SABC: strept-avidin-biotin complex; DAB: diaminobenzidine; ELISA: enzyme linked immunosorbent assay.

Table 3: The concentrations in brain tissue of TLR4, NFKB and TNFa ( $\bar{x} \pm \mathrm{s})$

\begin{tabular}{ccccc}
\hline Groups & n & TLR4 & NFkB & TNFa \\
\hline SO group & 5 & $14.26 \pm 2.08$ & $11.53 \pm 2.13$ & $12.34 \pm 2.25$ \\
IC group & 5 & $62.45 . \pm 7.26^{\Delta}$ & $75.16 \pm 9.12^{\Delta}$ & $67.78 \pm 8.37 \Delta$ \\
SA group & 5 & $34.28 \pm 5.04^{*}$ & $39.23 \pm 4.38^{*}$ & $35.16 \pm 4.71^{*}$ \\
PT group & 5 & $30.45 \pm 4.32^{* \#}$ & $31.64 \pm 4.46^{* \#}$ & $28.76 \pm 4.03^{* \#}$ \\
\hline
\end{tabular}

$\Delta P<0.01$, vs the SO group; ${ }^{*} P<0.01$, vs the IC group; $P>0.05$, vs the SA group. 


\section{Competing interests}

The authors declare that they have no competing interests.

\section{Authors' contributions}

GYL was responsible for design, experiment and manuscript writing. XXY, LQ LZ and DF carried out the experiments, data acquisition and analysis. All authors read and approved the final manuscript.

\section{Acknowledgements}

This study was supported by grant-in-aids for The National Natural Science Foundation of China (grant No. 30873391).

\section{Author Details}

'Institute of Cerebrovascular Diseases, Affiliated Hospital of Qingdao University Medical College, Qingdao 266003, China, 2Department of Emergency, Yantai Yuhuangding Hospital, Yantai 264000, China and ${ }^{3}$ Department of Neurology, Anhui Municipal Hospital, Hefei 230011, China

Received: 27 March 2010 Accepted: 9 July 2010

Published: 9 July 2010

\section{References}

1. Kaushal V, Schlichter LC: Mechanisms of microglia-mediated neurotoxicity in a new model of the stroke penumbra. J Neurosci 2008, 28:2221-2230

2. Caso JR, Pradillo JM, Hurtado O, Leza JC, Moro MA, Lizasoain I: Toll-like receptor 4 is involved in subacute stress-induced neuroinflammation and in the worsening of experimental stroke. Stroke 2008, 39:1314-1320

3. Bi X, Yan B, Fang S: Quetiapine regulates neurogenesis in ischemic mice by inhibiting NF-kappaB p65/p50 expression. Neurol Res 2009, 31:159-166.

4. Stuppner $\mathrm{H}$, Wagner $\mathrm{H}$ : New cucurbitacin glycosidesfrom Picrorhiza Kurrooa. Planta Medica 1989, 55:559-563.

5. Wang DQ, He ZD, Feng BS: Chemical constituents from Picrorhiza scrophulariflora. Acta Botanica Yunnanica 1993, 15:83-88.

6. Liu J, Liu BL, Zhang JQ, Zhang N: Hepatoprotective and choleretic action of Picrorhiza scrophulariae flora Pennell. Chin J New Drugs 2002, 11:459-461.

7. Li T, Liu JW, Zhang XD, Guo MC, Ji G: The neuroprotective effect of picroside 2 from hu-huang-lian against oxidtive stress. Am J Chin Med 2007, 35:681-691.

8. Liu JW, Yu YJ, Zheng PY, Zhang XD, Li T, Cao Y, Guo MC: Synergistic protective effect of picroside 2 and NGF on PC12 cells against oxidative stress induced by $\mathrm{H}_{2} \mathrm{O}_{2}$. Pharmacol Rep 2007, 59:573-579.

9. He LJ, Liang M, Hou FF, Guo ZJ, Xie D, Zhang X: Ethanol extraction of Picrorhiza scrophulariiflora prevents renal injury in experimental diabetes via anti-inflammation action. J Endocrinol 2009, 200(3):347-355

10. Li $P$, Matsunaga $K$, Yamakuni T, Ohizumi $Y$ : Potentiation of nerve growth factor-action by picrosides 1 and 2, natural iridoids, in PC12D cells. Eur J Pharmacol 2000, 406:203-208.

11. Li P, Matsunaga K, Yamakuni T, Ohizumi Y: Picrosides 1 and 2, selective enhancers of the mitogen-activated protein kinase-dependent signaling pathway in the action of neuritogenic substances on PC12D cells. Life Sci 2002, 71:1821-1835.

12. Tao YW, Liu JW, Wei DZ, Su W, Zhou WY: Protective effect of picroside 2 on the damage of culture PC12 cells in vitro. Chin J Clin Pharmacol Ther 2003, 8:27-30

13. Guo MC, Cao Y, Liu JW: Protective effects of picroside 2 on glutamate injury of PC12 cells. Chin J Clin Pharmacol Ther 2007, 12:440-443.

14. Yin JJ, Zhang W, Du F: Effects of extraction of Huhuanglian on apoptosis and $\mathrm{BCl}-2$ gene in penumbra area in ischemia reperfusion rats. Shandong J Traditil Chin Med 2005, 24:364-366.

15. Yang XW, Ji XM, Guo YL, Du F: Effect of rhizoma picrorhizae on nerve growth factor in rat brain following cerebral ischemia. Acta Acad Med Qingdao University 2008, 44:69-71.

16. Yan WJ, Li Z, Wang HP, Shen W, Du F: Picroside 2 inhibit apoptosis and expressions of iNOS following cerebral ischemic reperfusion injury in rats. Chin Pharmacol Bull 2009, 25:1677-1678.
17. $L i Z, X u X Y$, Shen $W$, Guo YL: The interferring effects of picroside 2 on the expressions of NF-KB and I-KB following cerebral ischemia reperfusion injury in rats. Chin Pharmacol Bull 2010, 26:52-56.

18. Li Q, Guo YL, Li Z, Xu XY: The interference of picroside 2 on the expressions of Caspase- 3 and PARP following cerebral ischemia reperfusion injury in rats. Chin Pharmacol Bull 2010, 26:342-345.

19. $L i Z$, Li Q, Guo YL, Qin $L H$, Luan $L$ J: The interference effect of picroside 2 on cerebral ischemia reperfusion injury in rats. Acta Anat Sinica 2010, 41:9-12.

20. Longa EZ, Weinstein PR, Carlson S, Cummins R: Reversible middle cerebral artery occlusion without craniectomy in rats. Stroke 1989, 20:84-91.

21. Gao HM, Zhang MZ: Asymmetry in the brain influenced the neurological deficits and infarction volume following the middle cerebral artery occlusion in rats. Behavioral and Brain Functions 2008 , 4:57-61.

22. Chinese Pharmcopoeia Commission: Chinese Pharmacopoeia, Part 1 2005 ed. Beijing: Chemical Industry Press; 2005:212-213.

23. Xiao YJ, Jiang ZZ, Yao JC, Huang X, Huang JF, Wang T, Zhang LY: Investigation of picroside 2's impacts on the P450 activities using a cocktail method. Chin J Nat Med 2008, 6:292-297.

24. Bederson JB, Pitts LH, Tsuji M, Nishimura MC, Davis RL, Bartkowski H: Rat middle cerebral artery occlusion: evaluation of the model and development of a neurologic examination. Stroke 1986, 17:472-476.

25. Hua F, Ma J, Ha T, Kelley JL, Kao RL, Schweitzer JB, Kalbfleisch JH, Williams $\mathrm{DL}, \mathrm{Li}$ C: Differential roles of TLR2 and TLR4 in acute focal cerebral ischemia reperfusion injury in mice. Brain Res 2009, 1262:100-108.

26. Yang QW, Li JC, Lu FL, Wen AQ, Xiang J, Zhang LL, Huang ZY, Wang JZ: Upregulated expression of toll-like receptor 4 in monocytes correlates with severity of acute cerebral infarction. J Cereb Blood Flow Metab 2008, 28:1588-1596

27. Boersma MC, Meffert MK: Novel roles for the NF-kappaB signaling pathway in regulating neuronal function. Sci Signal 2008, 1:pe7.

28. Lou HY, Wei XB, Zhang B, Sun X, Zhang XM: Hydroxyethylpuerarin attenuates focal cerebral ischemia-reperfusion injury in rats by decreasing TNF-a expression and NFKB activity. Acta Pharmaceutica Sinica 2007, 42:710-715.

29. Liu Y, Zhang XJ, Yang CH, Fan HG: Oxymatrine protects rat brains against permanent focal ischemia and downregulates NF-KB expression. Brain Res 2009, 1268:174-180

30. Webster CM, Kelly S, Koike MA, Chock VY, Giffard RG, Yenari MA: Inflammation and NF-KB activation is decreased by hypothermia following global cerebral ischemia. Neurobiol Dis 2009, 33:301-312.

31. Zhang HL, Gu ZL, Savitz SI, Han F, Fukunaga K, Qin ZH: Neuroprotective effects of prostaglandin $A$ in rat models of permanent focal cerebral ischemia are associated with nuclear factor-kappaB inhibition and peroxisome proliferator activated receptor-gamma up-regulation. $J$ Neurosci Res 2008, 86:1132-1141.

32. Li Z, Xu XY, Li Q, Zhang MZ, Shen W: Protective mechanisms of picroside 2 on aquaporin-4 expression in rat model of cerebral ischemia/ reperfusion injury. Neural Regen Res 2010, 5:411-417.

doi: 10.1186/1744-9081-6-43

Cite this article as: Guo et al., Anti-inflammation effects of picroside 2 in cerebral ischemic injury rats Behavioral and Brain Functions 2010, 6:43 